\title{
Kritische Sektoren anvisieren
}

\author{
Zu einer Entwarnung in der Umweltpolitik besteht aufgrund vieler persistenter \\ Umweltprobleme nach wie vor kein Anlass: Umweltpolitik muss deshalb in ihrem \\ Ansatz wirkungsvoller werden. Ein geeigneter Ansatzpunkt sind Sektorstrate- \\ gien, die Politikintegration weiter vorantreiben.
}

$\mathrm{K}$ Von Axel Volkery und Martin Jänicke limawandel, Verlust an biologischer Vielfalt, Bodendegradation oder Flächeninanspruchnahme: Dies sind nur einige persistente Umweltprobleme, bei denen über einen längeren Zeitraum hinweg keine signifikanten Trendverbesserungen feststellbar sind. Die rot-grüne Bundesregierung hat in den letzten Jahren zwar bereits viele Türen aufgestoßen Eine wirklich umweltentlastende Strategie erfordert aber mehr. In der neuen Legislaturperiode muss es um die generelle Marschrichtung einer nachhaltigen Entwicklung durch ökologische Modernisierung gehen. Als Schwerpunkte bieten sich an:

- eine unternehmensbezogene Innovationsstrategie, die - unter anderem über die Bildung von Lead-Märkten - zur ökologischen Modernisierung der Weltmärkte beiträgt;

- eine Neuorientierung der Staatstätigkeit in strategischen Politikfeldern, die nicht nur die ökologischen Innovationspotenziale relevanter Sektoren wie Energie, Bau oder Landwirtschaft gezielt erschließt, sondern auch zu einer den Faktor Arbeit umfassend entlastenden neuen Finanzpolitik beiträgt.

- Zudem ist ein weitreichender Handlungsbedarf durch europäische Rechtsvorgaben, etwa in der Chemie-, Produkt- oder Gewässerschutzpolitik, vorgegeben. Gleichfalls gilt dies für die Stärkung der institutionellen Strukturen internationaler Umweltpolitik (1).

\section{- Sektorpolitiken ökologisieren!}

Von wichtiger Bedeutung ist die Ökologisierung verursacherrelevanter Sektorpolitiken. Zwar wurde Umweltpolitik bereits in den siebziger Jahren als Querschnittsaufgabe definiert. Auch in der Amtszeit der rot-grünen Bundesregierung sind in vielen Bereichen, etwa mit dem Staatssekretärsausschuss für nachhaltige Entwicklung und der Nachhaltigkeitsstrategie, erste Schritte auf dem Weg einer sektorübergreifenden Behandlung von Umweltbelangen getätigt worden. Diese Maß- nahmen sind alleine aber noch nicht ausreichend. Notwendig ist eine umfassende „Umweltverträglichkeitsprüfung" der einzelnen Sektorpolitiken. Im Sinne einer Internalisierung der Problemverantwortung müssen negative Umwelteffekte der eigenen Tätigkeit und Alternativen von den betroffenen Ressorts identifiziert, Ziele und Maßnahmen konzipiert und in Abstimmung mit ihrem organisierten Umfeld umgesetzt werden. Dies schließt Berichtspflichten und Indikatoren der Erfolgskontrolle ein.

Dieser Typus von Sektorstrategien versucht, problembezogene Lernprozesse in den Fachministerien und bei ihren Politikadressaten zu stimulieren. Hierfür sind die Akteure gezielt mit den von ihnen (mit)verursachten Langzeitgefährdungen zu konfrontieren. Konsultationspflichten des Umweltbundesamtes oder des Bundesamtes für Naturschutz und regelmäßige Sektorkonferenzen, bei denen Ministerien und Interessenverbände Stellung zu Problemlagen nehmen und Lösungsvorschläge erarbeiten, sollten daher am Beginn des Prozesses stehen.

Da der Prozess der Strategieentwicklung in den Sektoren selbst nur zögerlich in Gang kommt, bedarf es einer zentralen Beauftragung durch Kabinett oder Parlament. Strategiebildung und -umsetzung müssen öffentlich kritisierbar sein. Die Bewertung dieses Prozesses setzt letztlich eine spezielle Institution voraus. Im Kanada ist beispielsweise ein Commissioner for Sustainable Development, der beim Rechnungshof angesiedelt ist, für die jährliche kritische Bewertung der Sektorstrategien zuständig.

Der Erfolg solcher Sektorstrategien ist von starken Umweltverwaltungen abhängig! Insbesondere bei der Beauftragung durch das Kabinett und bei der Festlegung der umweltpolitischen Prioritäten ist das Umweltressort gefragt. Es sollte daher in die Lage versetzt werden, Vorschläge für Politikinitiativen in anderen Politikbereichen auf die Kabinettsagenda setzen zu können. Zielgruppensteuerung dieser Art ersetzt allerdings keine allgemei- nen Regelungen, die die Rahmenbedingungen im Sinne einer generellen Tendenzsteuerung umweltbezogen verbessern. Dies gilt insbesondere für große und inhomogene Zielgruppen wie Verbraucher.

Da der Vollzug solcher Strategien weitgehend auf Landesebene liegt, wird hier in starkem Maße auf Verhandlungslösungen gesetzt werden müssen. Ein probates Mittel sind regelmäßige Bund-Länder-Ministerkonferenzen der Umweltminister und der Minister anderer Sektoren, die zu Lernprozessen auf beiden Seiten beitragen können.

Eine solche Umweltpolitik-Integration bringt letztlich eine Neuausrichtung ganzer Politikfelder. Im Bereich der Energiepolitik und in Ansätzen in der Agrarpolitik ist diese Lektion in Deutschland schon weitgehend verstanden worden. Die Verkehrspolitik ist angesichts der absehbaren Verkehrslawine im Transitland Deutschland ein weiterer wichtiger Kandidat. Und auch dem Baubereich bieten sich zahlreiche Ansatzpunkte, vom Null-Energiehaus über das Flächenrecycling bis hin zur Schaffung von „Brïcken für die Natur“. Die Bedeutung, aber auch die Schwierigkeit dieses Ansatzes der Umweltpolitikintegration erschließt sich am Beispiel des ehrgeizigen Ziels der Nachhaltigkeitsstrategie, die Flächeninanspruchnahme auf 30 Hektar pro Tag bis zum Jahr $2020 \mathrm{zu}$ senken. Dies erfordert nicht nur eine Revision der Förderung der auf Neubauten fixierten Eigenheimfinanzierung. Unter anderem sind auch eine umweltbezogene Reform der Grundsteuer bzw. der Einstieg in ein System handelbarer Flächennutzungsrechte auf kommunaler Ebene eine zentrale Erfolgsvoraussetzung.

\section{Anmerkung}

(1) In einem Gutachten für die Friedrich-Ebert- und die Heinrich-Böll-Stiftung sind hierfür von der Forschungsstelle für Umweltpolitik umfassende Ziel- und Maßnahmenvorschläge erarbeitet worden. Vgl. Jänicke, M./ Volkery, A.: Agenda 2002 ff. Perspektiven und Zielvorgaben nachhaltiger Entwicklung für die nächste Legislaturperiode. Berlin 2002 im Internet abrufbar unter www.fes.de oder www.boell.de

\section{Die Autoren}

Axel Volkery ist wissenschaftlicher Mitarbeiter, Prof. Dr. Martin Jänicke ist Leiter der Forschungsstelle für Umweltpolitik an der FU Berlin (FFU).

Kontakt: FFU, Ihnestr. 22, 14195 Berlin,

Tel. 030/ 838-56688, Fax -56685,

E-Mail: volkery@zedat.fu-berlin.de 
(c) 20I0 Authors; licensee IÖW and oekom verlag. This is an article distributed under the terms of the Creative Commons Attribution Non-Commercial No Derivates License (http://creativecommons.org/licenses/by-nc-nd/3.o/), which permits unrestricted use, distribution, and reproduction in any medium, provided the original work is properly cited. 